\title{
Dieta de um grupo de mico-leão-preto, Leontopithecus chrysopygus (Mikan) (Mammalia, Callitrichidae), na Estação Ecológica dos Caetetus, São Paulo ${ }^{1}$
}

Fernando de Camargo Passos ${ }^{2}$

\begin{abstract}
Diet of a black lion tamarin group, Leontopithecus chrysopygus (Mikan) (Mammalia, Callitrichidae), in Caetetus Ecological Station, São Paulo. In this study carried out in the Caetetus Ecological Station, São Paulo, southeastern Brazil, a wild group of black lion tamarins was accompanied during 1989 to 1991 , to analyse the dietary habits of the species. The scan sampling method was used to gather data. A total of 961 behaviors were recorded, of which feeding occupied $23.3 \%$ of the time involved in the behaviors. The major dietary components observed in the black lion tamarins were fruits, tree exudates and animal preys $(67.9 \%, 22.8 \%$ and $8.9 \%$ respectively). Fruits varied monthly from 47.4 to $97.1 \%$, being consumed more during the rainy season, while tree exudates varied from 0 to $54.7 \%$, and were consumed mainly during the dry season. The animal prey accounted for 0 to $15.8 \%$ of the diet. The most important dietary resource for the black lion tamarins was the fruit of Syagrus romanzoffiana Glass., representing $29.9 \%$ of the foraging observations. The diet exhibited pronounced differences among dry and rainy seasons, presumably as a consequence of the food shortage of fruits during dry season.

KEY WORDS. Callitrichidae, diet, feeding behavior, seasonal variation, black lion tamarin
\end{abstract}

O mico-leão-preto, Leontopithecus chrysopygus (Mikan, 1823), apresenta uma distribuição endêmica no Estado de São Paulo, estando entre as espécies mais ameaçadas do mundo (SEAL et al. 1990). Esta situação se deve à destruição quase total das florestas do Estado de São Paulo para a implementação de agricultura e pecuária. Há alguns anos atrás, pouco se conhecia a respeito da ecologia desta espécie, que chegou inclusive a ser considerada praticamente extinta (COIMBRA-FILHO 1970a, 1976). A espécie foi redescoberta no Parque Estadual do Morro do Diabo em Teodoro Sampaio em 1970 (CoIMBRA-FILHO 1970b). Mais recentemente foram realizados estudos sobre sua bionomia com o objetivo de subsidiar a preservação da espécie (CARVAlHO \& CARVAlHO 1989; CARVALHO et al. 1989; KEUROGHLIAN dados não publicados; VALLADARES-PÁDUA dados não publicados), havendo poucos estudos sobre a dieta.

1) Contribuição número 1150 do Departamento de Zoologia, Universidade Federal do Paraná.

2) Departamento de Zoologia, Universidade Federal do Paraná. Caixa Postal 19020, 81531-990 Curitiba, Paraná, Brasil.

E-mail: fpassos@bio.ufpr.br

Revta bras. Zool. 16 (Supl. 1): 269 - 278, 1999 
Existem diversos trabalhos abordando a dieta de várias espécies de Callitrichidae: Callithrix Erxleben, 1777 (RYLANDS 1986, 1989; FONSECA \& LACHER 1984; LACHER et al. 1981), Cebuella Gray, 1865 (RAMIREZ et al. 1977; SOINI 1993), Saguinus Hoffmannsegg, 1807 (PERES 1993; EgLER 1992; GARBER 1988; TERBORGH 1983), e Leontopithecus Lesson, 1840 (DIETZ et al. 1997; RYLANDS 1989, 1993). Poucas são as informações sobre dieta de Leontopithecus chrysopygus (COIMBRA-FilHo 1970b, 1976; CARVAlHO et al. 1989; MAMEDE-COSTA \& GODOI 1998).

Neste trabalho são apresentados dados quantitativos referentes à dieta do mico-leão-preto, indicando os tipos de alimentos consumidos, o comportamento utilizado na obtenção do alimento, e analisada a ecologia alimentar durante as estações seca e chuvosa, além de apresentar comparações com as outras espécies de Leontopithecus.

\section{MATERIAL E MÉTODOS}

Este estudo foi realizado na Estação Ecológica dos Caetetus $\left(22^{\circ} 15^{\prime}\right.$,

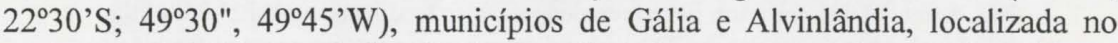
centro-oeste do Estado de São Paulo, Brasil. A área corresponde a um dos últimos fragmentos florestais do interior de São Paulo, apresentando vegetação do tipo floresta tropical semidecídua. O clima se caracteriza por uma estação seca que ocorre de abril a setembro, com precipitações mensais de 35 a $75 \mathrm{~mm}$ e temperaturas mais baixas, podendo ocorrer geadas, alternada com uma estação chuvosa de outubro a março, com 150 a $200 \mathrm{~mm}$ mensais de chuva e temperaturas mais elevadas. A floresta ocupa uma área de 2178 hectares, e se apresenta como um refúgio para a fauna silvestre.

No presente estudo um grupo de mico-leão-preto foi acompanhado de janeiro a junho de 1989 e entre maio de 1990 a janeiro de 1991, por meio de radiotelemetria. Os indivíduos do grupo foram marcados com colares de contas coloridas e tatuados. Na maioria das vezes o grupo foi observado desde o início de suas atividades, quando deixavam o oco utilizado como abrigo, sendo seguidos até sua retirada para o pernoite no mesmo oco ou em um outro, perfazendo um dia completo de atividade.

Este grupo de mico-leão-preto foi observado durante 53 dias, totalizando 217 horas de observações. No período de janeiro a junho de 1989 foi realizado um levantamento qualitativo da dieta do mico-leão-preto, enquanto no período de 1990/1991 as informações foram coletadas usando o método de amostragem instantânea "scan", com intervalos de 15 minutos (ALTMANN 1974). Foram registrados dados instantâneos referentes a hora, número de animais visíveis e atividade (categorizada como descanso, deslocamento, alimentação, forrageamento e outros). A atividade de forrageio foi considerada como o comportamento associado à procura para a obtenção de alimento de origem animal. Os registros de alimentação, quando um indivíduo manipulava ou ingeria alimento, foram coletados de maneira a diferenciar qual o tipo de alimento ingerido (frutos, exsudatos, presas, entre outros). No caso de frutos e exsudatos as plantas foram coletadas e herborizadas para posterior identificação por especialistas da Universidade Estadual de Campinas, onde as exsicatas foram depositadas como material testemunho. 


\section{RESULTADOS}

De um total de 961 registros comportamentais, a alimentação compreendeu $23,3 \%(\mathrm{~N}=224)$, o deslocamento $35,6 \%(\mathrm{~N}=342)$, o forrageamento $18,3 \%(\mathrm{~N}=176)$, o descanso $16,2 \%(\mathrm{~N}=156)$ e outros comportamentos $6,6 \%(\mathrm{~N}=63)$.

Ao todo trinta espécies vegetais foram utilizadas na dieta do mico-leão-preto, distribuídas por 21 famílias (Tab. I). Geralmente apenas a polpa dos frutos e as sementes são utilizadas e ingeridas, sendo a casca do fruto descartada, no caso das seguintes plantas: Protium spruceanum, Rhamnidium elaeocarpum, Struthanthus vulgaris, Celtis iguanae, C. pubescens, Cordia ecalyculata, C. superba e Mendoncia coccinea. Entretanto, algumas vezes o mico-leão-preto consome o fruto inteiro (Chrysophyllum gonocarpum), aproveita o sumo decorrente da mastigação (Ficus spp.), ou ainda roe o fruto, rejeitando a semente (Syagrus romanzoffiana).

Tabela I. Espécies vegetais utilizadas como alimento pelo mico-leão-preto, Leontopithecus chrysopygus, na Estação Ecológica dos Caetetus, Gália, São Paulo, Brasil. (EX) Exsudatos, $(F L)$ flores, (FR) frutos, (GA) galhas, $\left(^{*}\right)$ não amostrado no levantamento quantitativo.

\begin{tabular}{|c|c|c|c|c|}
\hline Familia & Espécie & Hábito & Dieta $(\%)$ & Item \\
\hline Anacardiaceae & Tapirira guianensis Aubl. & Árvore & 1,30 & FR, EX \\
\hline Annonaceae & Duguetia lanceolata St. Hill. & Árvore & * & FR \\
\hline Bignoniaceae & Stizophyllum perforatum Miers. & Liana & * & GA \\
\hline \multirow[t]{2}{*}{ Boraginaceae } & Cordia ecalyculata Vell. & Arvore & * & FR \\
\hline & Cordia superba Cham. & Arvore & * & FR \\
\hline Burseraceae & Protium spruceanum Benth. & Árvore & 1,30 & FR \\
\hline \multirow[t]{2}{*}{ Cactaceae } & Pereskia aculeata Mill. & Liana & $*$ & FR \\
\hline & Epiphyllum phyllanthus Haw. & Eplfita & 0,45 & $\mathrm{FL}$ \\
\hline Combretaceae & Terminalia sp. & Arvore & 1,80 & EX \\
\hline Euphorbiaceae & Croton floribundus Spreng. & Árvore & 0,45 & EX \\
\hline Loranthaceae & Struthanthus vulgaris Mart. & Epifita & 3,10 & FR \\
\hline Meliaceae & Trichilia catigua Juss & Arvore & $*$ & FR \\
\hline Mendonciaceae & Mendoncia coccinea Vell. & Liana & * & FR \\
\hline Menispermaceae & Abuta sp. & Liana & 4,00 & FR \\
\hline \multirow[t]{2}{*}{ Mimosaceae } & Inga marginata Will. & Arbusto & $\star$ & EX \\
\hline & Inga striata Benth. & Árvore & * & FR \\
\hline \multirow[t]{4}{*}{ Moraceae } & Ficus guaranitica Chodat & Árvore & 1,30 & FR \\
\hline & Ficus organensis Mic. & Árvore & * & FR \\
\hline & Ficus tomentella (Miq.) Miq. & Arvore & * & FR \\
\hline & Ficus trigona $\mathrm{L}$. & Arvore & 4,00 & FR \\
\hline \multirow[t]{2}{*}{ Myrtaceae } & Eugenia sulcata Spreng. & Arbusto & 0,45 & FR \\
\hline & Myrciaria sp. & Árvore & 6,70 & FR \\
\hline \multirow[t]{2}{*}{ Palmae } & Euterpe edulis Mart. & Ánore & 3,10 & EX \\
\hline & Syagrus romanzoffiana Glass. & Árvore & 29,9 & FR \\
\hline Rhamnaceae & Rhamnidium elaeocarpum Reiss. & Árvore & 3,10 & FR \\
\hline Rosaceae & Prunus selowii Koehne & Árvore & * & EX \\
\hline Rutaceae & Pilocarpus pauciflorus St. Hill. & Árvore & 15,60 & EX \\
\hline Sapotaceae & Chrysophyllum gonocarpum Engl. & Árvore & 2,20 & FR \\
\hline \multirow[t]{2}{*}{ Ulmaceae } & Celtis iguanae Sarg. & Liana & 7,10 & FR \\
\hline & Celtis pubescens H.B.K. & Liana & 4,50 & FR \\
\hline
\end{tabular}

Todos os frutos utilizados na dieta são do tipo carnoso e caracteristicamente zoocóricos, alguns dos quais são dispersos pelo próprio mico-leão-preto (PASSOS 1997). Os frutos que compõem a dieta foram consumidos preferencialmente maduros, embora em algumas ocasiões fossem utilizados ainda em fase de amadurecimento (Syagrus romanzoffiana). 
As presas consumidas incluíram desde invertebrados, principalmente artrópodes como insetos (baratas, besouros, esperanças e gafanhotos), até pequenos vertebrados como anfíbios anuros. $\mathrm{O}$ mico-leão-preto possui grande habilidade como predador, sendo suas presas capturadas e imobilizadas através de mordidas na cabeça, antes de serem consumidas. Anfíbios anuros são totalmente ingeridos, ao passo que as partes mais duras dos insetos podem ser descartadas.

O mico-leão-preto apresenta um vasto repertório comportamental para a captura de presas, as quais são encontradas e capturadas numa ampla variedade de substratos, como ocos e fendas em galhos e troncos, palmeiras, bambus, lianas, troncos em decomposição, pixídios de jequitibás [Cariniana estrellensis (Raddi) Kuntze], entre outros (PASSOS \& KEUROGHLIAN prelo).

Os exsudatos foram obtidos através de lambidas nas infrutescências que apresentavam gomas envolvendo os frutos (Euterpe edulis), ou utilizando gomas acumuladas nos troncos (Croton floribundus, Inga marginata, Pilocarpus pauciflorus, Prunus selowii, Tapirira guianensis e Terminalia sp.). Foi também observado o consumo de flor de uma cactácea epífita (Epiphyllum phyllanthus).

O grupo de micos foi observado alimentando-se de frutos em $67,9 \%$ do tempo (152 registros), de exsudatos em $22,8 \%$ (51), de presas em $8,9 \%$ (20), e de flores em $0,4 \%$ (1). O consumo de presas está subestimado em vista do método de amostragem de comportamento "scan sampling" ter sido realizado em intervalos de 15 minutos, uma vez que a captura, manipulação e ingestão é um evento realizado rapidamente, e muitas presas são consumidas entre o intervalo amostral. No decorrer do período amostrado, os frutos apresentaram uma variação mensal de $47,4 \%$ a $97,1 \%$ na dieta do mico-leão-preto, os exsudatos variaram de $0,0 \%$ a $54,7 \%$, as presas de $0,0 \%$ a $15,8 \%$, e as flores de $0,0 \%$ a $5,3 \%$ (Fig. 1 ).

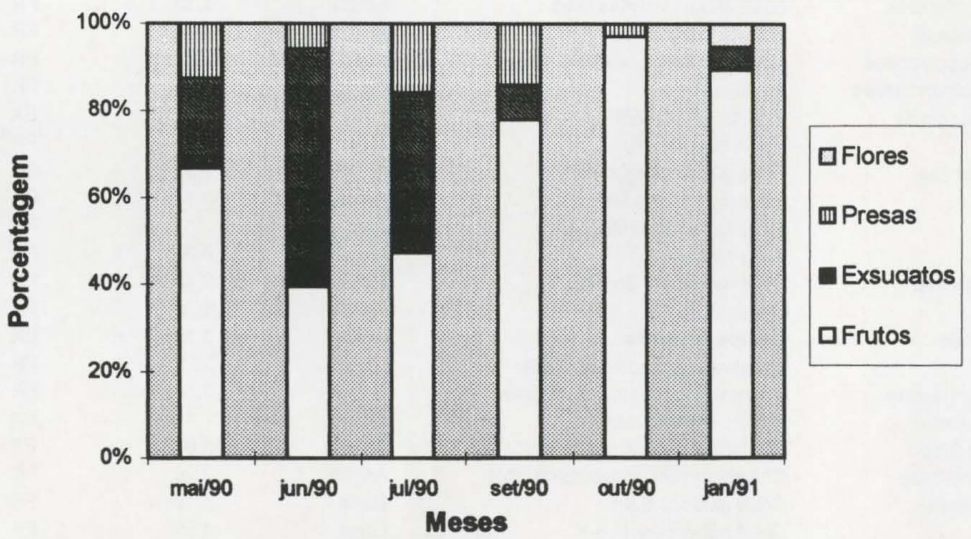

Fig. 1. Porcentagem de recursos utilizados mensalmente na dieta do mico-leão-preto, Leontopithecus chrysopygus, na Estação Ecológica dos Caetetus, Gália, São Paulo, Brasil.

A maior parte dos frutos consumidos pelo mico-leão-preto apresentou um período de frutificação curto, ao passo que algumas espécies (Celtis pubescens, $C$. iguanae, Chrysophyllum gonocarpum, Mendoncia coccinea e Syagrus romanzoffiana) apresentaram um longo período de frutificação e consumo (Tab. II). Embora 
os frutos de $C$. gonocarpum e $M$. coccinea tenham sido consumidos durante quatro meses, sua importância foi bem menor do que as outras três espécies, uma vez que poucos frutos foram consumidos em cada mês. Os frutos de $C$. pubescens, $C$. iguanae e $S$. romanzoffiana foram intensamente consumidos, com várias plantas destas espécies sendo visitadas a cada dia (apenas esses recursos foram responsáveis por $41.5 \%$ da dieta do mico-leão-preto e representaram $61.2 \%$ de todos os frutos consumidos no período). Dos recursos que forneceram exsudatos utilizados pelo mico-leão-preto destacaram-se Pilocarpus pauciflorus e Euterpe edulis, respectivamente utilizados em cinco e três meses, consumidos durante a estação seca (Tab. II).

Tabela II. Ocorrência mensal dos itens consumidos na dieta do mico-leão-preto, Leontopithecus chrysopygus, na Estação Ecológica dos Caetetus, Gália, São Paulo, Brasil. (EX) Exsudatos, (FL) flores, (FR) frutos, (GA) galhas.

\begin{tabular}{|c|c|c|c|c|c|c|c|c|c|c|c|}
\hline Espécies & Item & Jan & Fev & Mar & Abr & Mai & Jun & Jul & Ago & Set & Out \\
\hline Protium spruceanum & FR & $\oplus$ & & & & & & & & & \\
\hline Rhamnidium elaeocarpum & FR & $\oplus$ & & & & & & & & & \\
\hline Ficus organensis & FR & $\oplus$ & & & $\oplus$ & & & & & & \\
\hline Sthrutanthus vulgaris & FR & $\oplus$ & & & & & & & & $\oplus$ & \\
\hline Ficus trigona & $F R$ & $\oplus$ & & & & & & & & & $\oplus$ \\
\hline Inga striata & $F R$ & $\oplus$ & & & & & & & & & \\
\hline Pilocarpus pauciflorus & EX & $\oplus$ & & & & $\oplus$ & $\oplus$ & $\oplus$ & & $\oplus$ & \\
\hline Ficus tomentella & FR & & $\oplus$ & & & & & & & & \\
\hline Celtis pubescens & $F R$ & & $\oplus$ & $\oplus$ & $\oplus$ & $\oplus$ & & & & & \\
\hline Mendoncia coccinea & FR & & $\oplus$ & $\oplus$ & $\oplus$ & $\oplus$ & & & & & \\
\hline Cordia ecalyculata & $\mathrm{FR}$ & & & $\oplus$ & $\oplus$ & & & & & & \\
\hline Ficus sp. & $F R$ & & & & $\oplus$ & & & & & & \\
\hline Duguetia lanceolata & FR & & & & $\oplus$ & & & & & & \\
\hline Syagrus romanzoffiana & FR & & & & $\oplus$ & $\oplus$ & $\oplus$ & $\oplus$ & & $\oplus$ & $\oplus$ \\
\hline Celtis iguanae & FR & & & & $\oplus$ & $\oplus$ & $\oplus$ & & & & \\
\hline Tapirira guianensis & EX & & & & $\oplus$ & & $\oplus$ & & & & \\
\hline Stizophyllum perfuratum & GA & & & & $\oplus$ & & & & & & \\
\hline Cordia superba & $F R$ & & & & & $\oplus$ & & & & & \\
\hline Trichilia catigua & $F R$ & & & & & $\oplus$ & & & & & \\
\hline Chrysophyllum gonocarpum & FR & & & & & $\oplus$ & $\oplus$ & & & $\oplus$ & $\oplus$ \\
\hline Ficus guaranitica & FR & & & & & & $\oplus$ & & & & \\
\hline Abuta sp. & $F R$ & & & & & & $\oplus$ & & & & \\
\hline Euterpe edulis & EX & & & & & & $\oplus$ & $\oplus$ & & $\oplus$ & \\
\hline Inga marginata & EX & & & & & & & $\oplus$ & & & \\
\hline Terminalia sp. & EX & & & & & & & $\oplus$ & & & \\
\hline Croton floribundus & EX & & & & & & & & & $\oplus$ & \\
\hline Prunus selowii & EX & & & & & & & & & $\oplus$ & \\
\hline Pereskia aculeata & FR & & & & & & & & & $\oplus$ & \\
\hline Myrciaria sp. & $F R$ & & & & & & & & & & $\oplus$ \\
\hline Eugenia sulcata & FR & & & & & & & & & & $\oplus$ \\
\hline Presas & & $\oplus$ & $\oplus$ & $\oplus$ & $\oplus$ & $\oplus$ & $\oplus$ & $\oplus$ & & $\oplus$ & $\oplus$ \\
\hline
\end{tabular}

Os dois recursos alimentares mais importantes do período de estudo, com base no tempo gasto, foram os frutos de $S$. romanzoffiana e o exsudato de $P$. pauciflorus. Os frutos da palmeira S. romanzoffiana foram os recursos mais consumidos durante o período amostrado, com $29,9 \%$ do total da dieta e seus registros foram obtidos de abril até outubro. Esta espécie foi a mais importante da dieta do mico-leão-preto nos meses de julho (47,4\%), setembro $(58,0 \%)$ e outubro $(45,7 \%)$. Os frutos desta espécie também foram apanhados e consumidos no solo, principal- 
mente no mês de setembro. Este mês corresponde ao fim da estação seca, período em que esta espécie apresenta seus frutos completamente amadurecidos, muitos dos quais caídos no chão da floresta. $O$ exsudato de $P$. pauciflorus foi o principal recurso nos meses de estação seca, em maio $(33,4 \%)$ e junho $(41,5 \%)$, e compreenderam $15,6 \%$ do total da dieta. As presas, mesmo sub-amostradas, foram responsáveis por $8,9 \%$ da dieta, e em vista da grande quantidade de fragmentos de insetos presentes em quase todas as fezes, devem ser considerados importantes componentes da dieta do mico-leão-preto ao longo de todo o ano.

Os resultados mostram uma variação sazonal na dieta do mico-leão-preto (Tab. III). A base da dieta foi composta principalmente por frutos, consumidos em maior proporção durante a estação chuvosa $(94,3 \%)$, e menos utilizados na estação seca $(59,4 \%)$. Os exsudatos foram bem utilizados na estação seca $(29,4 \%)$, ao passo que na estação chuvosa estes recursos foram muito pouco explorados $(1,8 \%)$. As presas foram consumidas de forma mais acentuada nos meses de estação seca $(11,2 \%)$, sendo também pouco utilizadas na estação chuvosa $(1,9 \%)$. As diferenças na dieta entre as estações seca e chuvosa foram altamente significativas $\left(X^{2}=28.296\right.$, P.001).

Tabela III. Variação sazonal na dieta do mico-leão-preto, Leontopithecus chrysopygus, na Estação Ecológica dos Caetetus, Gália, São Paulo, Brasil.

\begin{tabular}{lcrr}
\hline Recursos & Estação seca & Estação chuvosa & Dieta (total) \\
\hline Frutos & $101(59,4 \%)$ & $51(94,3 \%)$ & $152(67,9 \%)$ \\
Exsudatos & $50(29,4 \%)$ & $1(1,9 \%)$ & $51(22,8 \%)$ \\
Presas & $19(11,2 \%)$ & $1(1,9 \%)$ & $20(8,9 \%)$ \\
Flores & $0(0,0 \%)$ & $1(1,9 \%)$ & $1(0,4 \%)$ \\
\hline Total de registros & 170 & 54 & 224 \\
H' trófica & 0,3972 & 0,1197 & \\
\hline
\end{tabular}

$X^{2}=28.296, \mathrm{P}<0.001 ;$ Teste " $\mathrm{t}$ " para $\mathrm{H}^{\prime}, \mathrm{t}=6.051, \mathrm{P}<0.025$.

\section{DISCUSSÃo}

As espécies de mico-leão, Leontopithecus spp., são primariamente insetívoras e frugívoras (COIMBRA-FILHO 1981; COIMBRA-FILHO \& MITTERMEIER 1977), variando sua dieta entre frutos, exsudatos, flores, insetos, aranhas e pequenos vertebrados. Os resultados deste estudo mostram que os frutos são os recursos predominantes da dieta $(67,9 \%)$ do mico-leão-preto na Estação Ecológica dos Caetetus, os quais são consumidos principalmente nos estratos intermediários da floresta (PASsos 1994). Para o mico-leão-de-cara-dourada, L. chrysomelas (Kuhl, 1820), os frutos representaram 74 a $89 \%$ da dieta (RYLANDS 1989).

O aproveitamento dos frutos não ocorre de modo igual no decorrer do ano. Os frutos são utilizados em alta proporção durante a estação chuvosa $(94,3 \%)$, quando apresentam maior disponibilidade. KEUROGHLIAN (dados não publicados) observou que no período mais úmido, entre os meses de novembro e dezembro, os frutos compreenderam $90 \%$ ou mais da dieta do mico-leão-preto.

O mico-leão-preto durante a estação seca utiliza-se de exsudatos com uma frequência significativamente maior que na estacão chuvosa $(29,4 \%$ e $1,9 \%$ da dieta, 
respectivamente), aparentemente como uma resposta à redução na oferta de frutos, cuja importância na dieta é reduzida na estação seca para 59,4\%.

Na floresta amazônica algumas espécies de primatas apresentam um padrão de dieta semelhante. Tanto Saguinus fuscicollis (Spix, 1823) quanto S. mystax (Spix, 1823) apresentam uma dieta dominada por frutos, porém durante o fim da estação chuvosa e início da estação seca, época de escassez de frutos, estes primatas se alimentam primariamente de exsudatos e néctar (PEREs 1993). Saguinus bicolor (Spix, 1823) também apresenta um consumo maior de exsudatos durante a estação seca e início da chuvosa (EGLER 1992).

A utilização de exsudatos é considerada uma forma alternativa de complementar as deficiências alimentares em épocas de escassez de alimento (RIZzINI \& COIMBRA-FILHO 1981; RYLANDS 1986), em vista da disponibilidade dos exsudatos ser menos sazonal do que a de outros recursos vegetais (LACHER et al. 1984). Durante estes períodos de baixa disponibilidade de frutos, muitas espécies de frugívoros apresentam mudanças na dieta, buscando fontes alternativas de recursos alimentares (FOSTER 1982; TERBORGH 1986).

Apesar das espécies de Saguinus e Leontopithecus não apresentarem as adaptações morfológicas e comportamentais de Callithrix e Cebuella, para o consumo de exsudatos, eles os consomem oportunisticamente. Os primatas do gênero Saguinus consomem exsudatos de um número limitado de famílias como Anacardiaceae, Combretaceae, Leguminosae e Vochysiaceae (GARBER 1993), ao passo que o mico-leão-preto foi observado consumindo exsudatos de sete famílias (Anacardiaceae, Combretaceae, Euphorbiaceae, Mimosaceae, Palmae, Rosaceae e Rutaceae).

Embora o mico-leão-de-cara-dourada, L. chrysomelas, tenha sido observado consumindo exsudato de vagens de Parkia pendula (Willd.) Benth. ex Walpers (RYLANDS 1989), o comportamento de utilizar gomas de vagens não foi observado na Estação Ecológica dos Caetetus (apesar de existirem espécies vegetais, como Inga Muller, que apresentam grandes quantidades de exsudatos em seus frutos). $\mathrm{O}$ comportamento normal é o de retirar o exsudato já acumulado nos troncos devido a danos naturais ou provocados por brocas de madeiras (GARBER 1993).

Foi surpreendente o alto consumo de exsudatos (22,8\% da dieta total) em comparação com outras espécies de Leontopithecus, causado provavelmente pelo ambiente mais sazonal, e portanto, pela menor disponibilidade de alimento durante a estação seca. O exsudato de Pilocarpus pauciflorus (Rutaceae) foi o segundo recurso mais importante da dieta, o que sugere a importância destes exsudatos na alimentação do mico-leão-preto, principalmente durante o período de baixa disponibilidade de frutos.

Uma resposta alternativa à redução na disponibilidade de frutos, encontrada nas outras espécies de Leontopithecus, é a utilização de néctar na dieta. O néctar de Symphonia globulifera Linnaeus é um importante recurso consumido pelo micoleão-dourado, L. rosalia (Linnaeus, 1766) (DIETZ et al. 1997), e pelo mico-leão-decara-dourada, L. chrysomelas (RYLANDS 1993).

Os frutos de gerivá, Syagrus romanzoffiana (Palmae), indiscutivelmente foram os recursos mais importantes da dieta do mico-leão-preto (29,9\% da dieta total), fato que já tinha sido observado por KEUROGHLIAN (dados não publicados) 
e MAMEDE-Costa \& Godol (1998). Provavelmente isto se deva ao longo período de frutificação desta espécie de palmeira (até sete meses), que apresenta frutos disponíveis a partir de abril, início da estação seca, prolongando-se até outubro, início da estação chuvosa. Sua frutificação ocorre durante toda a estação seca, quando é baixa a abundância de outras espécies de frutos. Embora o gerivá frutifique simultaneamente com outras espécies que representem recursos potenciais, o micoleão-preto parece se utilizar preferencialmente deste recurso. Além disso, essa espécie de palmeira se distribui por toda a reserva, ocorrendo em densidade relativamente alta e a disponibilidade de seus frutos por longos períodos favorece a manutenção da comunidade de frugívoros durante a estação seca.

Os micos-leões (Leontopithecus), são classificados como frugívoros-insetívoros, e os resultados mostram que o mico-leão-preto pode se enquadrar nessa classificação de frugívoro-insetívoro, mas também sazonalmente gomnívoro. Embora as presas tenham sido subestimadas devido ao método de observação utilizado e o intervalo amostral (15 minutos), o mico-leão-preto possui uma extraordinária versatilidade como predador (PASSOS \& KEUROGHLIAN prelo), capturando e ingerindo uma ampla variedade de presas, principalmente insetos e anuros. O papel de predador desempenhado pelos micos-leões já havia sido enfatizado por COIMBRAFILHO (1981).

A importância dos exsudatos na dieta desta espécie é muito maior do que em qualquer outra espécie de Leontopithecus. Aparentemente, estas diferenças estão relacionadas à sazonalidade mais acentuada das florestas semidecíduas do Estado de São Paulo, quando comparadas aos ambientes mais úmidos da Mata Atlântica onde ocorrem as outras espécies de mico-leão (RYLANDS 1993).

AGRADECIMENTOS. Agradeço ao Instituto Florestal de São Paulo e ao IBAMA por possibilitar que este estudo fosse realizado, a Cory Teixeira de Carvalho pela orientação e suporte sempre contínuo, a José Carvalho pela assistência no campo, a Hermógenes de Freitas Leitão-Filho pela identificação das espécies vegetais, a Anthony B. Rylands, Eleonore Setz, Ivan Sazima, Wagner A. Pedro, Wesley R. Silva pelos comentários e sugestões, a James J. Roper pela revisão do abstract, ao apoio da Universidade Estadual de Campinas (Programa de Pós-graduação em Ecologia) e ao financiamento da Capes e Fundação MB.

\section{REFERÊNCIAS BIBLIOGRÁFICAS}

ALTMANN, J. 1974. Observational study of behavior: sampling methods. Behaviour 49: 227-267.

Carvalho, C.T. DE \& C.F. DE Carvalho. 1989. A organização social dos sauís-pretos, (Leontopithecus chrysopygus Mikan), na reserva de Teodoro Sampaio, São Paulo (Primates, Callithricidae). Revta bras. Zool. 6 (4): 707-717.

Carvalho, C.T.; A.L.K. Albernaz \& C.A.T. LuCCA. 1989. Aspectos da bionomia do mico-leão-preto, Leontopithecus chrysopygus (Mikan, 1823) (Mammalia, Callitrichidae). Rev. Inst. Flor., São Paulo, 1 (1): 67-83.

COIMBRA-FILHO, A.F. 1970a. Considerações gerais e situação atual dos micos-leões escuros, Leontideus chrysomelas (Kuhl, 1820) e Leontideus chrysopygus 
(Mikan, 1823) (Callitrichidae, Primates). Rev. Brasil. Biol. 30 (2): 249-268. . 1970b. Acerca da redescoberta de Leontideus chrysopygus (Mikan, 1823) e apontamentos sobre sua ecologia (Callitrichidae, Primates). Rev. Brasil. Biol. 30 (4): 609-615.

- 1976. Leontopithecus rosalia chrysopygus (Mikan, 1823), o mico leão do Estado de São Paulo. Silvicultura 10: 1-36.

- 1981. Animais predados ou rejeitados pelo sauí-piranga, Leontopithecus r. rosalia (L., 1766) na sua área de ocorrência primitiva (Callitrichidae, Primates). Rev. Brasil. Biol. 41 (4): 717-731.

COIMBRA-FILHO, A.F. \& R.A. MitTERMEIER. 1977. Conservation of the brazilian lion tamarins (Leontopithecus rosalia), p. 59-94. In: PRINCE RAINIER \& G.H. BOURMNE (Eds). Primate Conservation. New York, Academic Press.

DieTZ, J.M.; C.A. PERES \& L. PINDER. 1997. Foraging ecology and use of space in wild golden lion tamarins (Leontopithecus rosalia). Amer. Jour. Primatol. 41: 289-305.

EGLER, S.G. 1992. Feeding ecology of Saguinus bicolor bicolor (Callitrichidae, Primates) in relict forest in Manaus, Brazilian Amazonia. Folia Primatol. 59: 61-76.

FonseCA, G.A.B. \& T.E. LACHER JR. 1984. Exudate-feeding by Callithrix jacchus penicillata in semideciduous woodland (Cerradão) in central Brazil. Primates 25 (4): 441-450.

FosTER, R.B. 1982. Famine on Barro Colorado Island, p. 201-212. In: E.D. LEIGH; A.S. RAND \& D.M. WINDSON (Eds). The ecology of a tropical forest: seasonal rythms and long-term changes. Washington, D.C., Smithsonian Institution Press, 468p.

GARBER, P.A. 1988. Diet, foraging patterns, and resource defence in a mixed species troop of Saguinus mystax and Saguinus fuscicollis in Amazonian Peru. Behaviour 105: 18-34.

1993. Feeding ecology and behaviour of the genus Saguinus, p.273-295. In: A.B. RYLANDS (Ed.). Marmosets and tamarins: systematics, behaviour, and ecology. Oxford. Oxford University Press.

LACHER, T.E. JR.; G.A.B. FonseCA; C. Alves JR. \& B. Magalhães-Castro. 1984. Parasitism of trees by marmosets in a central brazilian gallery forest. Biotropica 16: 202-209.

MAMEDE-CosTA, A.C. \& S. GodoI. 1998. Consuption of Syagrus romanzoffiana (Arecaceae) fruits, by blacklion tamarins (Leontopithecus chrysopygus) in south-eastern Brazil. Mammalia 62 (2): 310-313.

PAssos, F.C. 1994. Behavior of the black lion tamarin, Leontopithecus chrysopygus, in different forest levels in the Caetetus Ecological Station, São Paulo, Brazil. Neotropical Primates 2 (Suppl.): 40-41.

. 1997. Seed dispersal by black lion tamarin, Leontopithecus chrysopygus (Primates, Callitrichidae), in southeastern Brazil. Mammalia 61 (1): 109-111.

PASSOS, F.C. \& A. KEUROGHLIAN (prelo). Prey foraging behavior, microhabitats and techniques used by black lion tamarins, Leontopithecus chrysopygus Mikan 1823 (Mammalia, Callitrichidae). Revta bras. Zool.

Peres, C.A. 1993. Diet and feeding ecology of saddle-back (Saguinus fuscicollis) 
and moustached (S. mystax) tamarins in an Amazonian terra firme forest. Jour. Zool. 230: 567-592.

RAMIREZ, M.F.; C.H. FreESE \& C.J. ReVILHA. 1977. Feeding ecology of the pygmy marmoset, Cebuella pygmaea, in northeastern Peru, p.91-104. In: D.G. KLEIMAN (Ed.). The biology and conservation of the Callitrichidae. Washington, D.C., Smithsonian Institution Press, 354p.

RizZINI, C.T. \& A.F. CoIMBRA-FILHO. 1981. Lesões produzidas pelo sagui, Callithrix p. penicillata (E. Geoffroy, 1812), em árvores do cerrado (Callitrichidae, Primates). Rev. Brasil. Biol. 41 (3): 579-583.

RYLANDS, A.B. 1986. Ranging behavior and habitat preference of a wild marmoset group, Callithrix humeralifer (Callitrichidae, Primates). Jour. Zool. 210 (4): 489-514.

1989. Sympatric Brazilian callitrichids: the black-tufted-ear marmoset, Callithrix kuhli, and the golden-headed lion tamarin, Leontopithecus chrysopygus. Jour. Hum. Evol. 18: 679-695.

. 1993. The ecology of the lion tamarins, Leontopithecus: some intrageneric differences and comparisons with other callitrichids, p.296-313. In: A. B. Rylands (Ed.). Marmosets and tamarins: systematics, behaviour, and ecology. Oxford, Oxford University Press.

Seal, U.; J. Ballou \& C. Valladares-Padua. 1990. Leontopithecus Population Analysis. Apple Valley, MN: IUCN/SSC/CBSG.

SolNI, P. 1993. The ecology of the pygmy marmoset, Cebuella pygmaea: some comparisons with two sympatric tamarins, p.257-261. In: A.B. RYLANDS (Ed.). Marmosets and tamarins: systematics, behaviour, and ecology. Oxford, Oxford University Press.

TERBORGH, J. 1983. Five new world primates - a study in comparative ecology. Princeton, Princeton University Press, 260p.

1986. Keystone plant resources in the tropical forest, p.330-344. In: M.E. SOULÉ (Ed.). Conservation Biology: the science of scarcity and diversity. Suderland, Sinauer Associates Inc., 584p.

Recebido em 06.X.1999; aceito em 11.VI.1999. 